\title{
S3C6410-based CDMA Short Message Communication System on WINCE6.0 Platform
}

\author{
Linlin $\mathrm{Fu}^{1, \mathrm{a}}$, Jie Zhang $^{1, \mathrm{~b}}$, Shuying Cheng ${ }^{1, \mathrm{c}}$ \\ ${ }^{1}$ College of Physics and Information Engineering, and Institute of Micro-Nano Devices and Solar \\ Cells, Fuzhou University, Fuzhou, 350108, P.R. China \\ amysterygirllin@126.com , b13809507654@139.com , 'sycheng@fzu.edn.cn
}

Key words: S3C6410; AT commands; SMS; WINCE6.0; 3G Modem

\begin{abstract}
As a convenient, fast, reliable and economic value-added service, CDMA Short Message Service (SMS) becomes more and more popular in our daily work and lives. In this paper, the software package for sending and receiving short messages is realized, and it has simple interfaces and perfect functions. Based on WINCE 6.0, the USB port of Samsung S3C6410 is used as a virtual serial to realize the transmission of AT commands, and a specific realization of the processes and debugging results are given. The actual test results show that the software package can work stably. It can be used in the devices that support the WINCE 6.0.
\end{abstract}

\section{Introduction}

CDMA Short Message Service (SMS) is a service that provides a non-real-time transfer of messages with low-capacity and low-time performance, and realized through the CDMA network transmission ${ }^{[1]}$. As a cheap and convenient means of communication, SMS has become an indispensable part of people's life.

Previous work ${ }^{[2]}$ suggests that SMS based on $2 \mathrm{G}$ network is widely used in different fields. With the rapid development of the mobile communication, $3 \mathrm{G}$ communication has entered into people's life. The SMS based on $3 \mathrm{G}$ network is used in some areas, such as the monitoring ${ }^{[3]}$ and PDA PUSH Service ${ }^{[4]}$ and so on, but the research on this aspect is relatively less. In particular, there has been hardly any corresponding work of the SMS based on WINCE6.0 so far.

Based on the above, a software package is designed in this paper. Combining with $3 \mathrm{G}$ Modem and CDMA phone card, the USB Host of Samsung S3C6410 is used as a virtual serial interface to realize the communication between the microprocessor and the $3 \mathrm{G}$ Modem, and that between the system and the mobile phone. It can be used in the devices of supporting the WINCE 6.0, for example the remote monitoring system of the solar power station power.

\section{Realization of software package}

\subsection{Hardware platform}

As illustrated in Fig.1, the microprocessor S3C6410 is accessed to the CDMA network through wireless communication module. The hardware of the system consists of the embedded module and the CDMA communication module. The CDMA communication module is connected with S3C6410 through the USB host of the S3C6410.The CDMA communication module is composed of a $3 \mathrm{G}$ Modem and a phone card, and it is a regular mobile phone user for the communication company. 


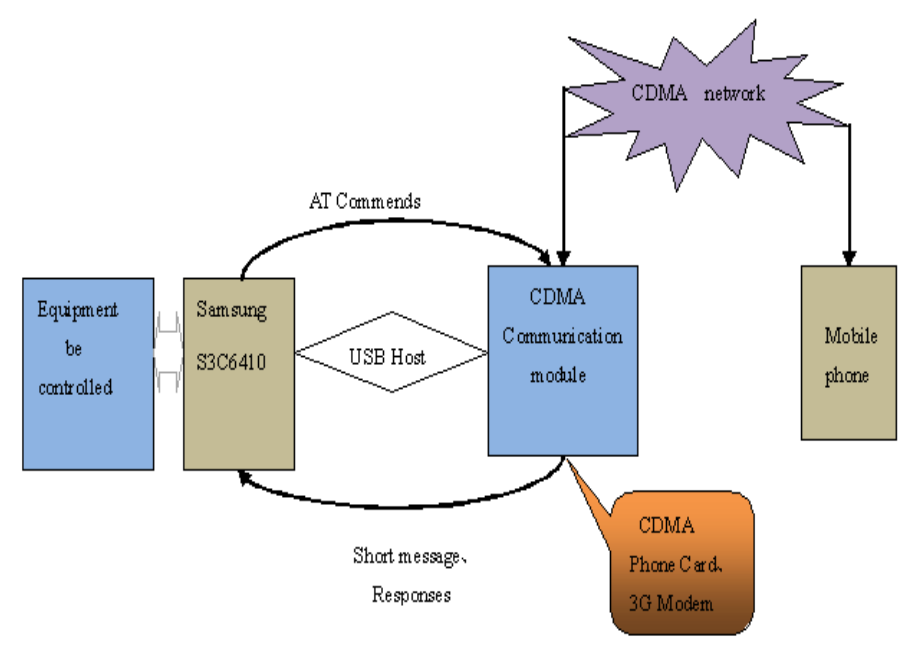

Fig.1 The hardware structure of the system

The embedded system can connect with the communication module through the USB port conveniently, and can control the communication module by AT commands ${ }^{[5]}$, read the short messages received by the module and analyze the commands through the program, thus it can realize not only the remote control of the system, but also the communication by sending the collected information to the designated mobile phone users.

\subsection{Software platform}

The software package was realized on WINCE6.0 platform. WINCE6.0 is an embedded operating system launched by the Microsoft office, which has a very good stability and real-time ${ }^{[6]}$. The AT instructions programming environment is VS2005, as a development environment of WINCE 6.0, VS2005 has a highly integration and expansibility.

\subsection{The composition of the software package}

CDMA message communication software package is mainly divided into five parts, the program structure is shown in Fig.2.

(1) serial port setting

Serial port settings include the opening and closing of the port.The virtual serial of the S3C6410 can communicate with the mobile phone after opening the serial, thus complete the operation of the communication module. The system will inquire if there are unreadable messages in the serial buffer when the serial is closing normally. If yes, then it will cancel the operation. It does not consider this kind of situation when mandatorily closed.

(2) SMS center number setting

The users can get and set the SMS center number. As a transfer station of the messages, the SMS center will be responsible for sending the messages to the specified destination.

(3) sending messages

After editing the message content and setting the phone number, we can call the SendMsg(num,sms) function to send the short messages to the designated phone users by clicking on the send button. 
(4) receiving messages

When a new message is arriving, it will be automatically stored. There will be a tip pointing out the location of the message. We can call the ReadMsg(int index) function to read the messages by inputting the index of the message according to the prompt. Then the message will be displayed in the test interface.

(5) error management

In order to improve the quality of the message communication. Error handling mechanism is added in the software package. When there are some errors in work process, the program will do the corresponding processing operation automatically. The operation process of the error handling mechanism is depicted in Fig.3.

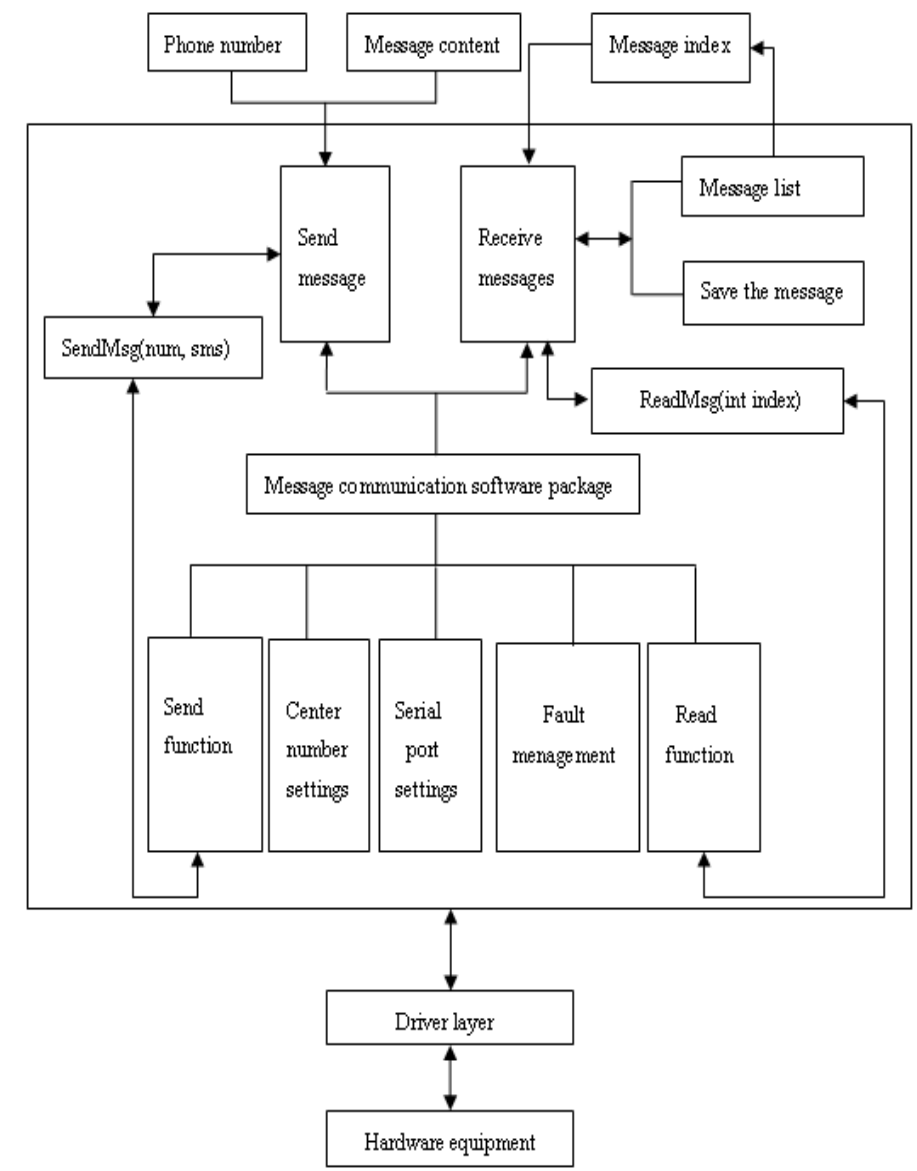

Fig.2 Message communication software package and interface

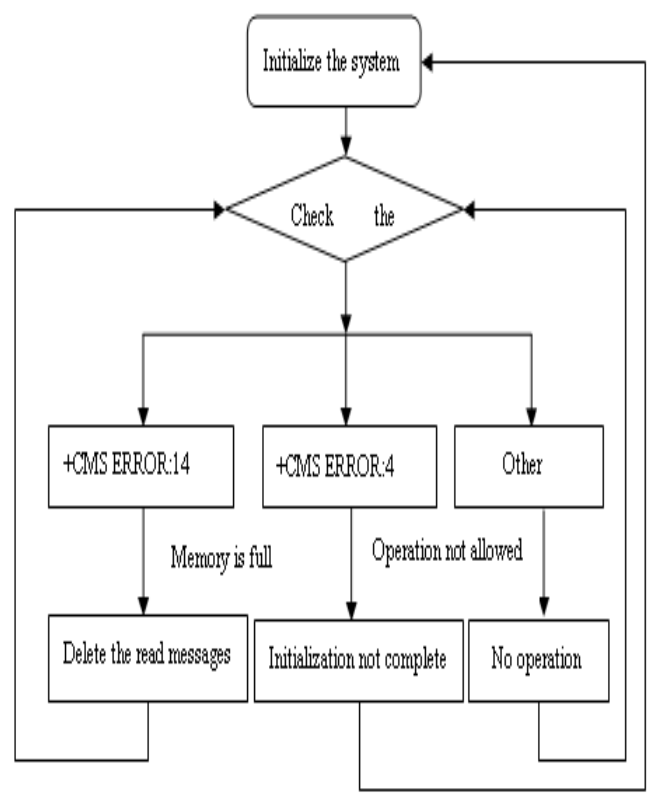

Fig.3 Error handling mechanism

\subsection{The operation process of the communication software}

In Fig.4, the operation process of the communication software is performed as follows:

Firstly, initialize the system, and send AT commends to test whether the hardware is connected ${ }^{[7]}$. Return "OK" if it is connected, otherwise return error information.

Then, open the serial to wait for the tasks, when sending messages, input the phone number and the message content, and call the send function to send the message by pressing the send button, return "OK" when sending successfully, and otherwise send again. When reading the message, input the index of the message according to the prompt, call the send function to send the read instruction to the serial. If reading successfully, the message content will be displayed in the test interface, or else read again. 
Finally, return to the waiting mode when there is no task.

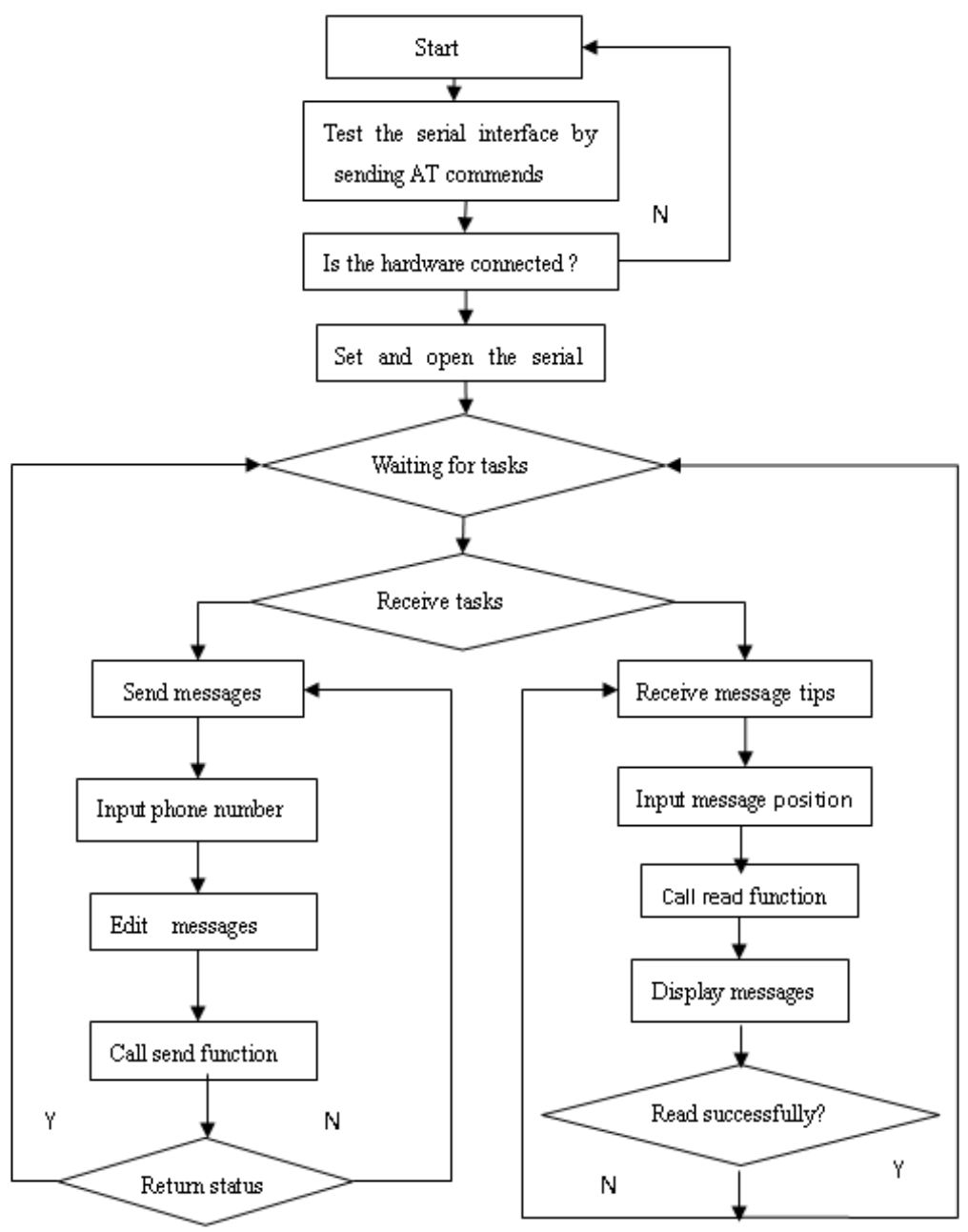

Fig.4 Operation process of the system

\section{Test Interface of the software package}

When the software package embedded in the device, according to a phone number and the message content, the device can send the message to the designated phone user.

Test interface is shown in Fig.5. Set the serial port and open the system, then input the phone number and the message content to be sent, last press the send button, the messages will be sent to the specified number (seen in Fig.6).

If there is a new message tip, the system will save the message automatically. When you want to read the message, input the index according to the tip and press the read button, the message will be displayed in the test interface. 


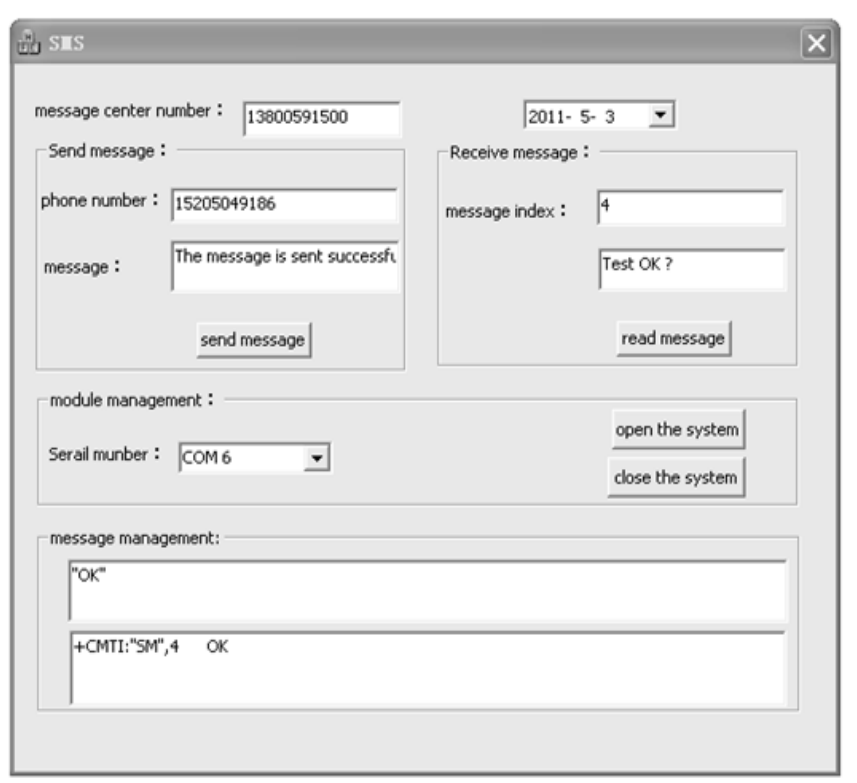

Fig.5 Test interface

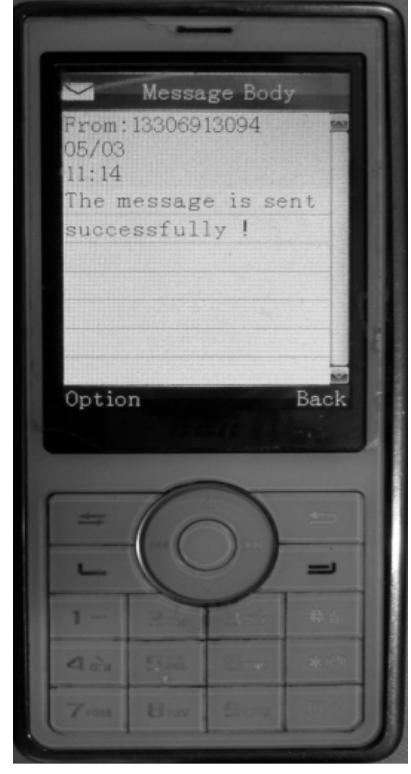

Fig.6 Mobile phone

\section{Conclusions}

Based on the AT instructions programming of VS2005, the USB port of the S3C6410 is used as a virtual serial port to realize the short message transceiver in the embedded system on the platform of WINCE 6.0. It is a simple and feasible method to realize the communication between the embedded system and the wireless network, and it can make up for the shortages of previous work on this aspect. It will make sense in theory and practice that this can be used in the remote monitoring and alarming system, there are some theoretical and realistic significance. Meanwhile, the software package is highly portable and can be applied to any device that supports WINCE.

\section{References}

[1] Terry S. Cheng, Kenneth W. Del Signore, Frances Lin, Cost effective treatment of short message service on the paging channel for the CDMA system, Bell Labs Technical Journal,12(2007)133-138.

[2] Jinqiu Xiao, Shumei Xu, Guohe Wu,Monitor system of the intelligent power earth lines based on GSM SMS protocol, ICEMI '09 .3(2009)178-179.

[3] Xiaoli Wang, Jianwei Li, CDMA-based application of wireless intelligent monitoring system, CMCE.4 (2010) 278-281.

[4] Jeong Kyoon Lee, Ki Young Lee, The Implementation of Security Message Protocol for PDA PUSH Service, TENCON 2005. (2007)1-4.

[5] Jichun Zhao, Junfeng Zhang Yufeng, Sufen Sun, Jian-xin Guo, The Design of Intelligence Supervisory System of Greenhouse Based on GPRS/3G,ICIS 2009.3(2009)17-18.

[6] Yulin Zhou, Yang Ning, Guiqiang Lu, Customization and Application Development of Windows CE.net Core, PHEI.(2005) 36-40.

[6] Douglas Boling, Programming Microsoft Windows CE.NET. third ed. Microsoft Press, America, 2003.

[7] Information on http : //en.wikipedia.org/ wiki/ SMS. 